\title{
The Relationship Between Cardiac Surgeon Experience and Patient Complexity: CA and NY statewide analysis
}

\author{
Gabe Weininger ${ }^{1}$, Arnar Einarsson ${ }^{2}$, Makoto Mori $^{3}$, Cornell Brooks ${ }^{1}$, Michael Shang ${ }^{1}$, \\ Roland Assi ${ }^{3}$, Prashanth Vallabhajosyula ${ }^{1}$, and Arnar Geirsson ${ }^{3}$ \\ ${ }^{1}$ Yale School of Medicine \\ ${ }^{2}$ Faculty of Medicine, School of Health Sciences, University of Iceland \\ ${ }^{3}$ Yale University School of Medicine
}

November 2, 2020

\begin{abstract}
Background: Complex cardiac operations may have better outcomes when performed by mid-career surgeons compared with surgeons in early or late stages of their career. However, it is unknown how cardiac case complexities are distributed among surgeons of different experience levels. Methods: We performed a cross-sectional study using New York (NY) and California (CA) statewide surgeon-level coronary artery bypass grafting (CABG) outcome data, including 336 cardiac surgeons who performed 43,604 CABGs. Surgeon-level data including observed mortality rate (OMR) and expected mortality rates (EMR) was collected from 2014-2016 in NY and 2015-2016 in CA. Surgeons' number of years-in-practice was determined by searching for each surgeon's training history on online registries. Loess and linear regression models were then used to characterize the relationship between surgeon EMR and surgeon years-in-practice. Results: The median number of surgeon years-in-practice was 20 (interquartile range [IQR] 11-28) with median case volume 103 (IQR 42,171). The median surgeon observed to expected mortality (O:E) ratio was 0.87 (IQR 0.19-1.4). Linear regression relating EMR to years in practice showed that EMR was similar across years in practice. Linear regression relating surgeon isolated CABG O:E ratio to years in practice also showed similar outcomes across years in practice. Conclusion: There is a relatively equal distribution of high and low risk CABG cases among surgeons of differing experience levels. This equal distribution of high and low risk cases does not reflect a triaging of more complex cases to more experienced cardiac surgeons, which prior research shows may optimize patient outcomes.
\end{abstract}

\section{Introduction}

Several surgeon-related factors are known to be associated with surgical outcomes. Surgeon years in practice is one of these factors, with some reports suggesting a concave relationship on complex cases, in which outcomes of early-career surgeons improve for the first number of years in practice, stabilize for the middle of their career, and finally decline for very late career surgeons ${ }^{1-3}$. Such findings may suggest the importance of triaging cases of different complexity to surgeons of different experience levels. However, it remains unknown if the composition of cases in terms of patient risk profile changes with surgeons' number of years in practice.

There have been very few studies on this topic, however the little evidence that exists in general surgery points to earlier-career surgeons receiving more challenging and higher-risk cases ${ }^{4-6}$. In cardiac surgery, a prior study showed that surgeons with lower risk-adjusted mortality rates tended to perform CABG surgery on higher risk patients ${ }^{7}$. Additionally, it has been shown that experienced surgeons with 9-17 years in practice may perform best on highly complex reoperative cases ${ }^{1}$. However, it is unknown if complex cases are distributed in a way that would optimize patient care in light of this observed trend. The relationship between cardiac surgeon experience and case complexity remains unclear in a field with high risk operations that may warrant further examination. 


\section{Methods}

\section{Data source}

The Yale institutional review board waived approval and the need for patient consent since the datasets are publicly available. The data was obtained from the publicly available 2014-2016 New York State Cardiac Data Reporting System and 2015-2016 California Report on CABG Surgery ${ }^{8,9}$. Both of these states have mandatory public reporting systems for surgeon-level cardiac surgery outcome data. We collected observed mortality rate (OMR) and expected mortality rate (EMR) for isolated CABG in both states. EMR is calculated from multivariable risk models developed by the New York State Department of Public Health and California CABG Outcomes Reporting Program, both of which account for various patient demographics and comorbidities ${ }^{8,9}$. Some of these demographics and comorbidities include ejection fraction, previous MI, cardiogenic shock, previous cardiac surgery or PCI, renal failure, liver disease, peripheral vascular disease, endocarditis, BMI, and others ${ }^{8,9}$. Based on these multivariable risk models, EMR captures the average risk profile of a surgeon's cases. Operative mortality for both states is defined as death within 30-days from surgery or within the index hospitalization.

We determined surgeons' number of years in practice by collecting each surgeon's training history from The Cardiothoracic Surgery Network (CTSnet, ctsnet.org). Each surgeon's final year of schooling was subtracted from 2016 (the latest year captured in the New York and California data) to determine number of years in practice. For surgeons whose training history was not listed on CTSnet, we searched other online resources including the website of the surgeon's current hospital and healthgrades.com. International medical graduates (IMGs) were excluded from the study because IMGs may have practiced as surgeons overseas, which may have obscured the actual years in practice. We combined surgeon-level outcomes for individual surgeons practicing at multiple hospitals.

\section{Statistical analysis}

We examined the association between surgeon-level EMR and surgeon years in practice via linear regression models. To account for differences in the model specifications used for California and New York multivariable risk models, EMRs were standardized by dividing surgeon EMR by the corresponding state's average mortality rates for isolated CABG, $1.67 \%$ for New York and $2.44 \%$ for California. This yielded standardized risk relative to each state's average observed mortality rate. A sensitivity analysis was also performed to ensure EMR was not skewed by surgeons with low case volumes. Observed to expected (O:E) ratios were calculated for each surgeon and analyzed via linear regression with years in practice, with adjustment for case volume. For this O:E ratio regression, case volumes were divided by the average CABG case volumes for the New York and California study periods to account for the longer study period captured in the New York data. We defined statistical significance as $p<0.05$. All data analysis was performed in $\mathrm{R}$ version 3.6.3 (R Foundation for Statistical Computing).

\section{Results}

There were 366 surgeons who performed 43,604 isolated CABG that met the inclusion criteria for our study (Table 1). Of these, 252 surgeons operated in California and 114 operated in New York. The median number of years-in-practice was 20 (interquartile range [IQR] 11-28) and the median O:E ratio for isolated CABG was 0.87 (IQR 0.19-1.4). Years in practice was roughly normally distributed. The median EMR and case volume for California surgeons were 2.42 (IQR 1.76, 2.95) and 78.5 (IQR 32.25-129.5) respectively. For New York surgeons median EMR and case volume were 1.44 (IQR 1.22, 1.68) and 158 (IQR 87.75-243.5).

Linear regression relating normalized EMR to years in practice did not show a statistically significant relationship, with similar EMR across the number of years in practice (Table 2). This trend was also visually summarized using Loess regression (Figure 1). A sensitivity analysis in which surgeons with $<25$ cases were excluded to determine if these surgeons were skewing the data also showed no statistically significant relationship between years in practice and EMR (Table 3). Lastly, linear regression relating surgeon isolated CABG O:E ratio to years in practice, with adjustment for surgeon case volume, also showed no statistically 
significant relationship with similar O:E ratios across the number of years in practice (Table 4). This trend was also visually summarized using Loess regression (Figure 2).

\section{Discussion}

Using two statewide datasets from two high-volume states, we showed that CABG case complexities are evenly distributed across cardiac surgeons of various experience levels. This is important for several reasons. First, this shows that in cardiac surgery there is a relatively equal distribution of high and low risk patients among surgeons of differing experience levels. This stands in contrast to the findings in other specialties, such as The Cognitive Changes and Retirement among Senior Surgeons Study (CCRASS), which found that surgeons self-reported an increase in case volume and a decrease in case complexity over time ${ }^{4}$. The CCRASS was supported by a study which showed that general surgeons with fewer than 15 years in practice operated on patients of higher pre-operative risk ${ }^{5}$. Another study of vascular surgeons showed that surgeons within their first 5 years of practice had a greater proportion of nonelective cases with a higher degree of comorbidities and larger aneurysms ${ }^{10}$. To our knowledge, this is the first time this relationship between surgeon experience and average patient risk profile has been investigated in cardiac surgery.

Determining whether there is a proportionate distribution of high and low risk cases for surgeons of all experience levels is valuable, both for the sake of training and for the sake of patient care. Ensuring early career surgeons see cases of different preoperative risk and complexity is likely to be an important feature of comprehensive competency. On the other hand, some reports have shown that middle to late career surgeons obtain the best outcomes on complex operations ${ }^{1,3}$, which may argue for a distribution of cases in which the more experienced surgeons receive more of the high-risk cases to optimize patient outcomes.

Our data also demonstrated that there is no statistically significant relationship between risk-adjusted outcomes on isolated CABG and surgeon experience. This is a valuable independent finding for two reasons. It demonstrates that early-career surgeons demonstrate competency in CABG surgery, which has been challenged in some studies ${ }^{11,12}$ and supported by others ${ }^{13,14}$. Secondly, some articles have discussed whether there should be a maximum age at which surgeons are allowed to practice ${ }^{15,16}$. It used to be the case in the United Kingdom that surgeons could not perform surgery for the Public Health Service past the age of 65. Our data suggests that this surgeon age-maximum is not necessary, as late-career surgeons did not demonstrate inferior outcomes to early-career surgeons on isolated CABG surgery.

\section{Strengths and Limitations}

The strengths of our study include the use of two high-volume statewide databases, which capture a very large number of surgeons relative to many other studies that have investigated this relationship. Additionally, our dataset is comprised of two mandatory statewide outcome reporting systems, which minimize potential selection bias or a narrow scope of payer-specific databases such as Medicare data. A limitation of our dataset is that while EMR is a strong measure of high vs low risk patients, it does not necessarily reflect information about case complexity or whether a case is elective vs nonelective, which will be an important area for future investigation. Differences in the California and New York risk models is another limitation of our dataset - though these differences should be adequately adjusted for by normalizing EMR by respective state mortality averages.

\section{Conclusions}

CABG cases of differing pre-operative risk are distributed relatively equally among surgeons of different experience levels. This relatively equal distribution of high and low risk cases stands in contrast to findings in other specialties and does not demonstrate a triaging of more complex cases to more experienced cardiac surgeons, which prior research shows may optimize patient outcomes.

\section{Conflicts of Interest and Funding}

There were no conflicts of interest and this study was not funded.

\section{References}


1. Moon MR, Henn MC, Maniar HS, et al. Impact of Surgical Experience on Operative Mortality Following Reoperative Cardiac Surgery. Ann Thorac Surg . Published online June 3, 2020.

2. Prystowsky JB. Are Young Surgeons Competent to Perform Alimentary Tract Surgery? Arch Surg . 2005;140(5):495-502.

3. Duclos A, Peix J-L, Colin C, et al. Influence of experience on performance of individual surgeons in thyroid surgery: prospective cross sectional multicentre study. BMJ . 2012;344.

4. Lee HJ, Drag LL, Bieliauskas LA, et al. Results from the cognitive changes and retirement among senior surgeons self-report survey. J Am Coll Surg . 2009;209(5):668-671.e2.

5. Guidry CA, Newhook TE, Turrentine FE, Sohn M-W, Sawyer RG, Jones RS. Observations on Surgeons' Case Selection, Morbidity, and Mortality Following Board Certification. Ann Surg . 2016;263(3):487492.

6. Walch G, Bacle G, Lädermann A, Nové-Josserand L, Smithers CJ. Do the indications, results, and complications of reverse shoulder arthroplasty change with surgeon's experience? J Shoulder Elbow Surg . 2012;21(11):1470-1477.

7. Glance LG, Dick A, Mukamel DB, Li Y, Osler TM. Are High-Quality Cardiac Surgeons Less Likely to Operate on High-Risk Patients Compared to Low-Quality Surgeons? Evidence from New York State. Health Serv Res . 2008;43(1 Pt 1):300-312.

8. New York State Department of Health. Cardiovascular Disease Data and Statistics. 2019. https://www.health.ny.gov/statistics/diseases/cardiovascular/. Accessed: July 1, 2019.

9. Coronary Artery Bypass Graft: Surgeon Performance Ratings, 2015-2016. OSHPD.

10. Arnaoutakis DJ, Scali ST, Neal D, et al. Surgeon experience association with patient selection and outcomes after open abdominal aortic aneurysm repair. J Vasc Surg . Published online February 27, 2020.

11. Chai C-Y, Chen C-H, Lin H-W, Lin H-C. Association of increasing surgeon age with decreasing inhospital mortality after coronary artery bypass graft surgery. World $J$ Surg . 2010;34(1):3-9.

12. Maruthappu M, Gilbert BJ, El-Harasis MA, et al. The Influence of Volume and Experience on Individual Surgical Performance: A Systematic Review. Annals of Surgery . 2015;261(4):642-647.

13. Hartz AJ, Kuhn EM, Pulido J. Prestige of training programs and experience of bypass surgeons as factors in adjusted patient mortality rates. Med Care . 1999;37(1):93-103.

14. Choudhry NK, Fletcher RH, Soumerai SB. Systematic review: the relationship between clinical experience and quality of health care.Ann Intern Med . 2005;142(4):260-273.

15. Blasier RB. The Problem of the Aging Surgeon: When Surgeon Age Becomes a Surgical Risk Factor. Clin Orthop Relat Res . 2009;467(2):402-411.

16. Bunkin IA. When Does a Surgeon Retire? JAMA . 1983;250(6):757-758.

Tables/Figures (6/10)

Table 1: Surgeon Characteristics

\begin{tabular}{llll}
\hline & Total $(\mathrm{N}=366)$ & California $(\mathrm{N}=252)$ & New York $(\mathrm{N}=114)$ \\
\hline Years in Practice & & & \\
$\quad$ Mean (SD) & $20.02(10.62)$ & $19.96(10.9)$ & $20.17(10.03)$ \\
$\quad$ Median (Q1, Q3) & $20(11,28)$ & $20(11,28)$ & $20(12,28)$ \\
$\begin{array}{l}\text { Isolated CABG Cases } \\
\quad \text { Mean (SD) }\end{array}$ & $119.14(100.33)$ & $91.6(71.81)$ & $180.02(125.01)$ \\
$\quad$ Median (Q1, Q3) & $103(42,171)$ & $78.5(32.25,129.5)$ & $158(87.75,243.50)$ \\
Isolated CABG EMR & & & \\
$\quad$ Mean (SD) & $2.2(1.1)$ & $2.50(1.17)$ & $1.51(0.43)$ \\
$\quad$ Median (Q1, Q3) & $2.01(1.45,2.7)$ & $2.42(1.76,2.95)$ & $1.44(1.22,1.68)$ \\
\hline
\end{tabular}

$\mathrm{CABG}=$ coronary artery bypass graft $\mathrm{EMR}=$ expected mortality rate $\mathrm{IQR}=$ interquartile range 
The table summarizes surgeon characteristics for all CABG surgeries performed in New York between 20142016 and California between 2015-2016.

Table 2: Regression Model for Surgeon EMR on Isolated CABG

\begin{tabular}{llll}
\hline & Normalized EMR & Normalized EMR & Normalized EMR \\
\hline Predictors & Estimates & $C I$ & $p$ \\
Years in practice & 0.00 & -0.00 to 0.01 & 0.092 \\
Observations & 366 & 366 & 366 \\
\hline
\end{tabular}

$\mathrm{CABG}=$ coronary artery bypass graft $; \mathrm{EMR}=$ expected mortality rate

The table shows results of a linear regression model.

Table 3: Sensitivity Analysis using Surgeons with $>25$ Cases

\begin{tabular}{lll}
\hline & Normalized EMR for Surgeons with $>\mathbf{2 5}$ Cases & Normalized EMR for Surgeons with $>$ 25 \\
\hline Predictors & Estimates & $C I$ \\
Years in Practice & 0.00 & -0.00 to 0.01 \\
Observations & 308 & 308 \\
\hline
\end{tabular}

$\mathrm{EMR}=$ expected mortality rate

The table shows results of a sensitivity analysis that used only surgeons from our dataset with $>25$ cases.

Table 4: Regression Model for Surgeon O:E Ratio on Isolated CABG

\begin{tabular}{llll}
\hline & CABG O:E Ratio by Years in Practice & CABG O:E Ratio by Years in Practice & CABG \\
\hline Predictors & Estimates & $C I$ & $p$ \\
Years in Practice & 0.02 & -0.00 to 0.05 & 0.094 \\
Case Volume & -0.00 & -0.01 to -0.00 & $\mathbf{0 . 0 3 8}$ \\
Observations & 366 & 366 & 366 \\
\hline
\end{tabular}

$\mathrm{CABG}=$ coronary artery bypass graft; $\mathrm{EMR}=$ expected mortality rate; $\mathrm{O}: \mathrm{E}$ Ratio=observed to expected mortality ratio

The table shows the results of a linear regression model relating surgeon $\mathrm{O}: \mathrm{E}$ ratio on isolated $\mathrm{CABG}$ to years in practice and case volume.

Figure 1: Relationship between cardiac surgeon years in practice and EMR on isolated CABG

Histogram of years in practice for cardiac surgeons who performed isolated coronary artery bypass grafting (CABG) surgery between 2014-2016 in New York and 2015-2016 in California (Panel A). The relationship between normalized expected mortality rate (EMR) and years in practice is visualized using Loess regression (Panel B). Shaded regions on Panel B represent a 95\% confidence interval.

Figure 2: Relationship between cardiac surgeon years in practice and O:E ratio on isolated CABG

The relationship between surgeon observed to expected mortality (O:E) ratio on isolated CABG and years in practice is visualized using Loess regression. Shaded regions represent a $95 \%$ confidence interval. 

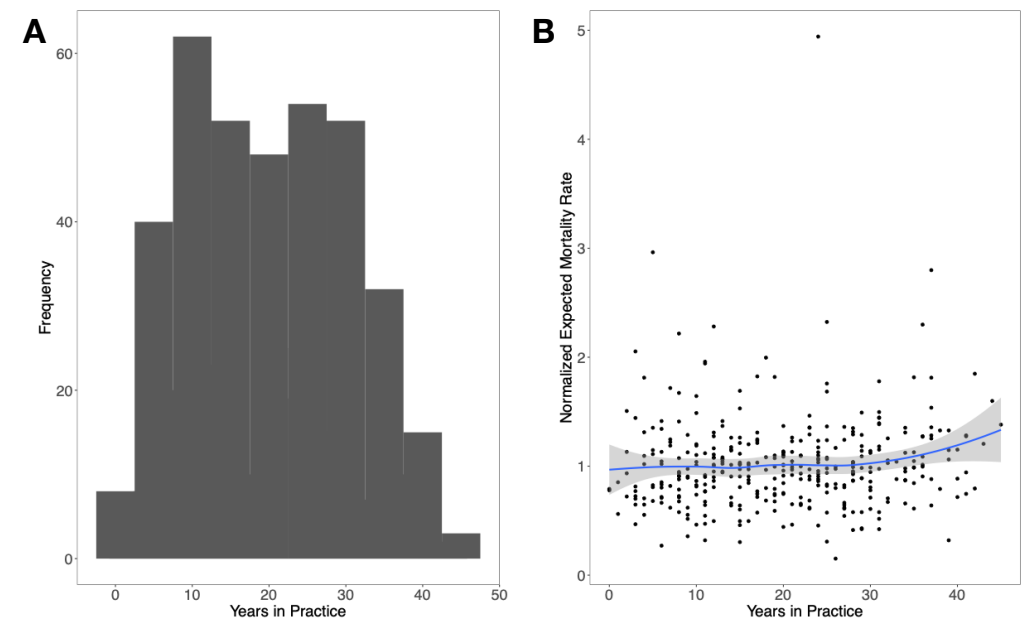


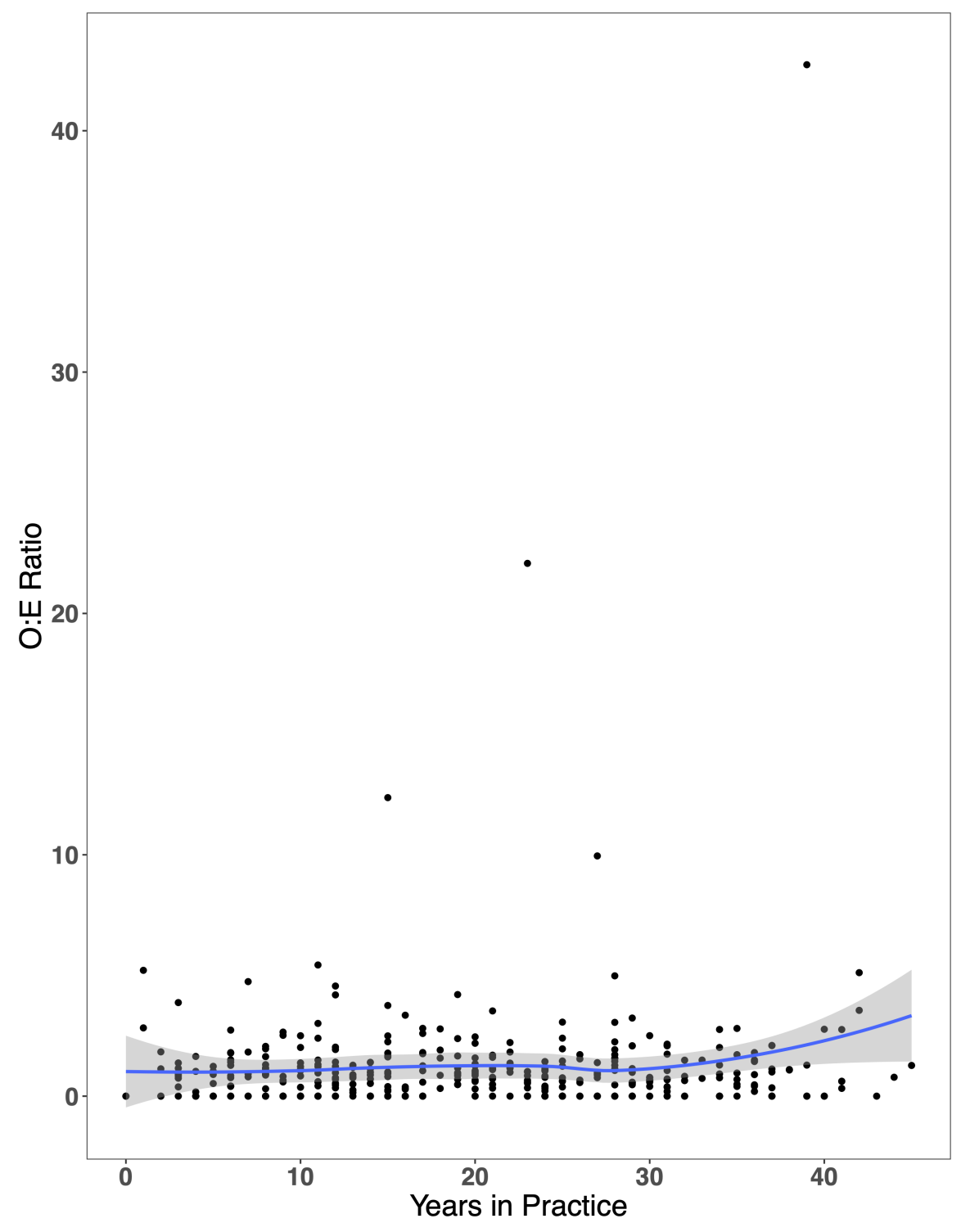

